Boll. Accademia Gioenia di

Scienze Naturali - Catania
BOLLAG Vol. 52, N. 382 (2019)

Difesa ecosostenibile delle colture agricole pp. DECA22 - DECA28

ISSN 0393-7143

Anno di fondazione 1824

\title{
La gestione della lotta ecosostenibile contro i fitofagi dannosi: il caso della Tignola del pomodoro ${ }^{\dagger}$
}

\author{
Gaetano Siscaro [1] \\ Dipartimento di Agricoltura, Alimentazione e Ambiente, Università degli Studi di Catania
}

\begin{abstract}
Riassunto
La Tignola del pomodoro Tuta absoluta, fitofago invasivo di primario interesse economico in Italia, può infestare le piante di pomodoro durante tutto il ciclo di produzione. Specie di origine neotropicale, è da molti anni presente in Sud America e a partire dal 2006 si è rapidamente insediata anche nel bacino del Mediterraneo. Più di recente la sua diffusione ha interessato l'Africa e l'Eurasia divenendo una seria minaccia per la produzione di pomodoro a livello globale sia in serra che in pieno campo. Vengono illustrate le più significative strategie di controllo integrato (IPM) che comprendono l'adozione di misure di prevenzione, strumenti di monitoraggio e metodi di controllo sostenibile a ridotto impatto eco-tossicologico. In serra, queste strategie possono essere adottate sia prima del trapianto (rimozione dei residui colturali, tecniche igienico-sanitarie, utilizzo di reti anti-insetto) che dopo (uso di feromoni, protezione e potenziamento dei nemici naturali indigeni, applicazioni razionali di insetticidi e bioinsetticidi). Tra le applicazioni di controllo biologico, vengono illustrati e discussi il ruolo del miride predatore Nesidiocoris tenuis e le tecniche per la sua valorizzazione.
\end{abstract}

Parole chiave: Tuta absoluta, controllo integrato, colture protette, entomofagi, miridi predatori

\section{Sustainable pest management in cropping systems: the case study of the South American tomato pinworm}

\section{Summary}

The South American tomato pinworm, Tuta absoluta is a tomato key pest in Italy infesting the crop during the whole production cycle. This Neotropical species has been present in South America for many years and since 2006 it has rapidly established in the Mediterranean basin. More recently, the pest is spreading in Africa and Eurasia becoming a global threat for tomato crops in greenhouse and open field. Integrated pest management strategies (IPM) including prevention measures, monitoring and sustainable control methods with low eco-toxicological impact are reported. Before crop transplanting they consist in the removal of crop residues, use of insect-proof nets and sanitization methods; after the transplanting, pheromone based technics, protection and enhancement of native natural enemies and sustainable applications of insecticides and bio-insecticides are the best options. Among the methods of biological control, the role of the predator Nesidiocoris tenuis and the techniques for its successful exploitation are discussed.

Key words: Tuta absoluta, integrated pest management, protected crops, natural enemies, predatory mirids

\footnotetext{
${ }^{\dagger}$ Nota presentata nell'Adunanza pubblica del 15 novembre 2018

*e-mail: gsiscaro@unict.it
} 


\section{Introduzione}

Tuta absoluta (Meyrick) (Lepidoptera: Gelechiidae) è una specie di origine neotropicale (Fig.1), la cui diffusione è rimasta limitata al Sud America fino alla metà dello scorso decennio. Descritta sulla base di un singolo esemplare di sesso maschile rinvenuto sulle Ande peruviane, è stata successivamente segnalata in numerosi altri paesi sudamericani, in molti dei quali viene riportata quale fitofago di primario interesse a partire dagli anni ' 80 .
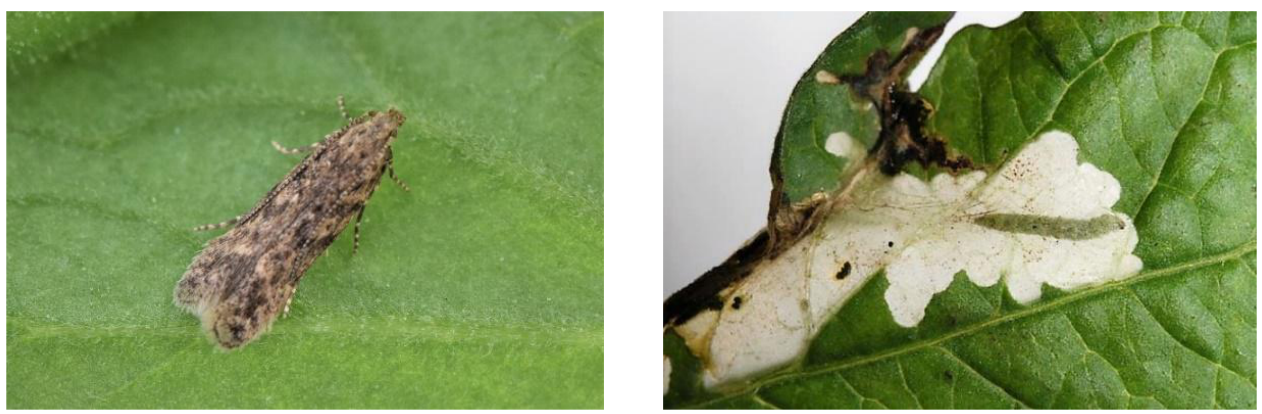

Figura 1 - Tuta absoluta: adulto (a sinistra, foto A. Biondi) e larva (a destra, foto G. Siscaro) vista in trasparenza all'interno di una mina fogliare.

La prima segnalazione al di fuori dell' area di origine è avvenuta nel 2006 in Spagna e da allora ha colonizzato rapidamente il bacino del Mediterraneo, invadendo pressoché tutto il continente europeo, l'Africa, il Medio Oriente e l'Eurasia spingendosi fino all'India, costituendo di fatto una minaccia per i principali paesi produttori di pomodoro al mondo, Cina e USA compresi $[1,2]$. Analisi genetiche hanno confermato che le popolazioni presenti nelle nuove aree d'invasione sono geneticamente uniformi e provengono da un unico ceppo sud americano [3].

Il fitofago può infestare tutti gli organi epigei delle piante di pomodoro inducendo rilevanti danni economici sia in pieno campo che in serra e in tutta la filiera di produzione e commercializzazione. Alla dannosità diretta deve sommarsi anche il maggiore costo dovuto all'incremento degli interventi insetticidi. Si stima che nel caso in cui il fitofago dovesse invadere anche aree geografiche come Cina e Stati Uniti d'America sarà interessata quasi il 90\% della produzione globale di pomodoro. Da ciò deriva la sua rilevanza fitosanitaria e il notevole interesse che la comunità scientifica rivolge verso questa specie [3].

Trattasi di una specie polivoltina, che svolge diverse generazioni in un anno e presenta un'elevata capacità riproduttiva. Le uova sono deposte, per lo più singolarmente, su foglie e steli; le larve neonate si introducono nei tessuti della pianta formando delle gallerie di dimensioni crescenti, all'interno delle quali si alimentano fino a maturità (Fig. 1).

Le larve incrisalidano principalmente nel terreno e gli adulti presentano un'attività di tipo crepuscolare [2]. La distribuzione sui vari organi della pianta varia in funzione dello sviluppo e della fenologia della pianta stessa. Infatti, le larve e le mine tendono a localizzarsi prevalentemente nelle foglie espanse nella parte mediana della chioma, mentre le uova e quindi le larve giovani sono più frequenti nella parte apicale.

Oltre al pomodoro T. absoluta può infestare altre solanacee sia coltivate (melanzana, peperone, patata e tabacco) che spontanee, come Solanum nigrum L. [2]. La disponibilità di piante ospiti alternative facilita la permanenza del fitofago nelle aree interessate anche in assenza della coltura.

Considerato che le strategie di controllo attualmente adottate sia nei paesi d'origine che in quelli di più recente invasione sono incentrate sul controllo chimico, il rischio di effetti secondari legati alla insorgenza di popolazioni resistenti agli insetticidi e agli effetti indesiderati su organismi utili è elevato $[3,4,5]$. Proprio nel tentativo di contenere questi rischi, diverse tecniche $\mathrm{e}$ strategie di controllo eco-sostenibili, alternative all'applicazione di insetticidi convenzionali, sono state sviluppate negli ultimi anni o sono in corso di valutazione.

In questa nota si illustrano le strategie di controllo ecosostenibili più efficaci o più promettenti applicate nel bacino del Mediterraneo e in Italia per il contenimento di questo fitofago. 


\section{Strategie di controllo ecosostenibile}

Per le sue caratteristiche biologiche e la rilevanza fitosanitaria, unitamente alla forte tendenza a sviluppare popolazioni resistenti agli insetticidi, questa specie è considerata un key-pest del pomodoro sia in pieno campo che in coltura protetta. In serra può essere efficacemente contenuta applicando correttamente tecniche di controllo integrato di tipo sia preventivo che curativo [6].

Misure preventive, da adottare anche prima del trapianto della coltura, prevedono la rimozione dei residui colturali, l'applicazione di procedure di sanitizzazione, l'uso di materiale di propagazione indenne, la messa in opera e la manutenzione degli apprestamenti serricoli per la protezione fisica. Con la coltura in atto le misure di protezione attiva prevedono l'impiego di feromoni per il monitoraggio e la cattura massale, la protezione e il potenziamento dei nemici naturali indigeni e l'applicazione di insetticidi e bioinsetticidi a ridotto impatto eco-tossicologico.

Di seguito, vengono descritte le peculiarità e le modalità di applicazione di queste tecniche.

\subsection{Prevenzione}

L'individuazione di potenziali nemici naturali e le strategie per la loro conservazione e il loro potenziamento, sono elementi prioritari per l'attuazione di programmi di controllo di qualsiasi fitofago esotico, come appunto la Tignola del pomodoro. Numerose specie di parassitoidi e predatori indigeni sono stati segnalati su T. absoluta nel bacino del Mediterraneo; questi limitatori naturali, rapidamente adattatisi al nuovo fitofago, rivestono un ruolo cruciale nel contenimento delle popolazioni di questa specie invasiva. Essendo questi antagonisti non specifici, possono riprodursi a carico di altri ospiti alternativi presenti su flora spontanea. La loro abbondanza in una determinata area è quindi fortemente influenzata alla presenza di piante spontanee e colture sempreverdi che possano garantire una sufficiente biodiversità funzionale.

La rimozione e la tempestiva distruzione dei residui della precedente coltura è fondamentale per eliminare una potenziale fonte d'inoculo per le nuove coltivazioni. Inoltre, tra le altre pratiche agronomiche, si raccomanda di adottare adeguate rotazioni colturali e impiegare materiale vivaistico privo di infestazioni. Considerata la possibile permanenza nel terreno di crisalidi di T. absoluta, un intervento di solarizzazione del terreno può costituire un valido strumento di contenimento del fitofago unitamente all'installazione di trappole cromotropiche gialle o nere possibilmente attivate con feromone sessuale per la cattura massale di adulti già presenti all'interno della serra. Tra le misure volte a limitare l'introduzione di adulti all'interno delle serre occorre prestare particolare attenzione alla corretta applicazione e manutenzione di reti anti-insetto con idoneo ordito alle aperture e di doppie porte agli ingressi [6].

\subsection{Monitoraggio}

L'acquisizione di informazioni sull' andamento dell'infestazione è un elemento essenziale per l'applicazione di strategie di controllo efficaci. Anche se attualmente in Italia non sono disponibili soglie economiche d'intervento nei confronti di T. absoluta, i trattamenti insetticidi devono sempre essere supportati da adeguati schemi di monitoraggio che sono fondamentalmente basati sull'uso di trappole a feromone sessuale, associati al monitoraggio visivo su pianta. Questi strumenti agevolano l'individuazione della presenza di stadi più suscettibili e aiutano a contenere il numero degli interventi insetticidi [6].

\subsection{Misure di controllo}

\subsubsection{Confusione/disorientamento sessuale}

Grazie alla sintesi chimica del feromone sessuale di $T$. absoluta, sono disponibili sul mercato diffusori feromonici per applicazioni di tecniche di disturbo dell'accoppiamento in coltura protetta. Valutazioni sperimentali, realizzate in Italia utilizzando varie tipologie di diffusori [6], hanno dimostrato che tale tecnica risulta utile per abbassare la densità di popolazione del fitofago, purché 
applicate in serre adeguatamente isolate. Tuttavia, il ritrovamento nel Mediterraneo di popolazioni partenogenetiche (deuterotoche) della tignola presenti in natura [7], potrebbe avere implicazioni negative sull'efficacia di queste applicazioni biotecniche.

\subsubsection{Cattura massale}

Tre tipologie di trappole basate su sistemi attrattivi di tipo feromonico, luminoso e misto possono essere impiegate per la cattura massale degli adulti di T. absoluta; queste vengono collocate ad inizio coltura a livello pianta in ragione di $2-3 / 1000 \mathrm{~m}^{2}$. Ce ne sono disponibili in commercio vari modelli, e sono anche diffuse in campo molte varianti di fattura artigianale. Molto usate risultano trappole a funzionamento "misto" tipo feromoni/acqua ovvero feromoni/luce/acqua che collocate sia all'interno che all'esterno delle serre assicurano elevati livelli di catture di adulti di entrambi i sessi [6].

Alcune di queste trappole luminose, ad accensione automatica, permettono di ottenere elevati livelli di cattura all'interno della serra. L'efficacia di questi dispositivi in ambiente protetto è comunque strettamente vincolata alle condizioni d'isolamento della serra in modo da ridurre l'accesso di adulti dall'esterno della struttura. Più di recente sono stati messi a punto altri dispositivi per la cattura massale che utilizzano non solo l'attrattivo feromonico ma anche la componente cromoattrattiva: si tratta di fasce collanti di colore giallo, nero o trasparenti, innescate con il feromone sessuale di T. absoluta; queste possono essere collocate all'interno delle serre per diversa lunghezza.

\subsubsection{Controllo biologico}

Lo sviluppo di tecniche di controllo biologico rappresenta uno dei più promettenti strumenti per la gestione sostenibile di questo fitofago, grazie all'individuazione di diversi antagonisti naturali indigeni e alla disponibilità di efficaci agenti microbiologici quali funghi, nematodi e in particolare batteri. I formulati a base del batterio Bacillus thuringiensis Berliner (Eubacteriales: Bacillaceae) sono gli insetticidi microbiologici più utilizzati [3]. Applicati da decenni, questi bioinsetticidi sono considerati ecosostenibili, in quanto innocui per gli organismi superiori e altamente compatibili con l'uso di antagonisti naturali [5].

Un gran numero di nemici naturali è stato segnalato in associazione con T. absoluta, sia nelle aree di origine che in quelle di nuova introduzione. Il parassitoide oofago Trichogramma pretiosum Riley (Hymenoptera: Trichogrammatidae), in Sud America, e i miridi predatori Macrolophus pygmaeus (Rambur) e Nesidiocoris tenuis (Reuter) (Hemiptera: Miridae), in Europa e nel bacino del Mediterraneo, risultano in atto gli entomofagi più diffusi. Questi vengono impiegati mediante rilasci aumentativi o mediante strategie di controllo biologico conservativo nel contesto di programmi di controllo integrato (IPM) $[3,8,9]$.

Anche se in misura minore rispetto a quanto verificato in Sud America, dove sono stati segnalati oltre un centinaio di predatori e parassitoidi associati a T. absoluta, anche in Europa e in particolare in Italia sono state segnalate diverse specie di imenotteri parassitoidi larvali afferenti alle famiglie degli Eulofidi, Ichneumonidi e Braconidi [9]. Tra questi il più promettente e diffuso è Necremnus tutae Ribes \& Bernardo (Hymenoptera: Eulophidae) (Fig. 2), che è allevato in biofabbrica e rilasciato in colture protette anche in associazione con trattamenti microbiologici a base di B. thuringiensis.

Tra i predatori, i Miridi Diciphini N. tenuis, M. pygmaeus e Dyciphus errans (Wolff) sono considerati gli antagonisti più efficaci e diffusi nel bacino del Mediterraneo. In particolare $N$. tenuis, predatore generalista e zoo-fitofago (Fig. 2), già da diversi anni impiegato per il controllo di aleirodi, acari e tripidi in serra, si è dimostrato particolarmente attivo nei confronti delle uova e dei primi stadi larvali di T. absoluta [3, 9]. 

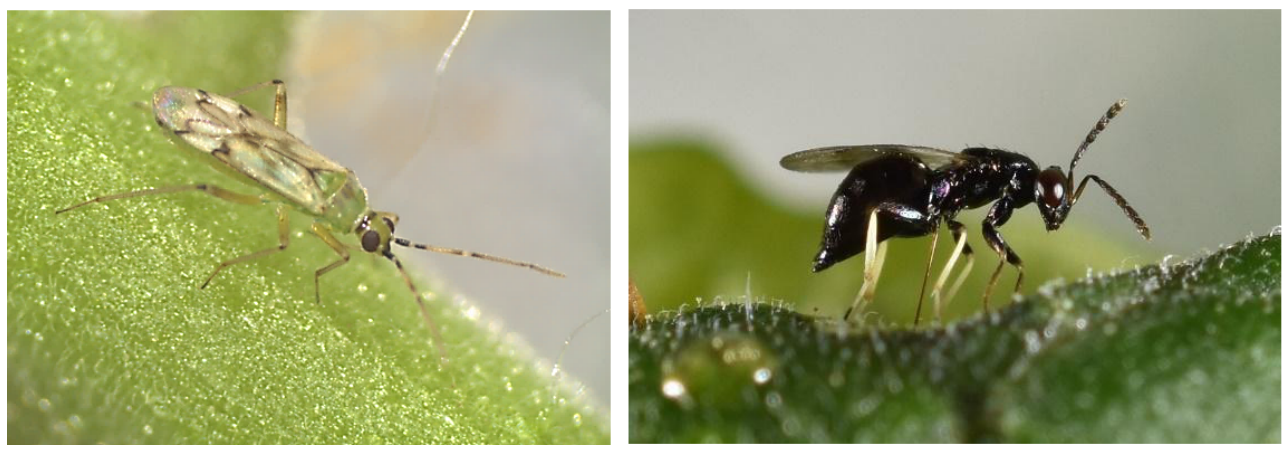

Figura 3 - adulto di Nesidiocoris tenuis (a sinistra, foto G. Siscaro) e femmina di Necremnus tutae (a destra, foto A. Biondi).

Come già detto, l'integrazione di rilasci inoculativi di $N$. tenuis con trattamenti microbiologici a base di $B$. thuringiensis risulta una strategia efficace, anche se occorre adattare l'intervento alle condizioni colturali. Tuttavia, in condizioni di scarsità di prede e nei periodi più caldi si possono osservare danni su pomodoro (in particolare anellature necrotiche degli apici) causati dall'attività trofica del miride [10]. Il ruolo di alcune piante alternative al pomodoro è attualmente oggetto di studio per la gestione del predatore e il contenimento dei suoi danni $[11,12]$. Inoltre, nuove conoscenze sul ruolo di questo predatore nella gestione dei fitofagi potranno derivare dalla recente scoperta della sua capacità, attraverso le punture di alimentazione, di induzione di meccanismi metabolici di difesa nelle piante [13].

\subsubsection{Controllo chimico}

Sia nelle aree geografiche d'origine che in quelle di nuova introduzione il controllo di T. absoluta è affidato in maniera preminente agli insetticidi [3, 4]. Questa situazione ha determinato ripercussioni negative sulla gestione del controllo integrato dei fitofagi nelle colture ortive, quali l'insorgenza di fenomeni di resistenza e l'impatto negativo su organismi utili. In Europa, diversi principi attivi sono stati registrati per il controllo di T. absoluta; tuttavia, gli insetticidi più utilizzati appartengono a quattro classi chimiche distinte con diverse modalità di azione, vale a dire diamidi, avermectine, spinosine e ossadiazine [4].

Tra gli insetticidi in atto autorizzati in Italia, quelli con un profilo eco-tossicologico ritenuto più favorevole sono: azadiractina, abamectina, B. huringiensis kurstaki, B. thuringiensis aizawai, clorantraniliprole, emamectina benzoato, indoxacarb, spinosad. Tutti questi formulati esplicano la loro azione sui vari stadi larvali del fitofago con modalità e tempistiche differenti. Oltre a questi principi attivi di tipo convenzionale, è stato osservato che lo zolfo elementare in polvere ha un'azione antideponente nei confronti delle femmine [14]. Interessanti sviluppi potranno inoltre derivare dalle recenti sperimentazioni condotte su alcuni bioinsetticidi di origine vegetale, quali gli olii essenziali di agrumi [15]. Nel complesso sono disponibili 16 principi attivi insetticidi che agiscono mediante 13 diversi meccanismi di azione tossica nei confronti di T. absoluta [4].

Sono noti da diversi anni fenomeni di resistenza agli insetticidi sia in Sud America che in Europa; la specie si è dimostrata in grado di sviluppare rapidamente ceppi capaci di detossificare principi attivi appartenenti a diversi gruppi chimici, quali organofosfati, spinosine, avermectine, chitino-inibitori (diflubenzuron), oxadiazine (indoxacarb), diamidi (clorantraniliprole). Considerato l'interesse che assume la questione a livello globale, la presenza di popolazioni resistenti o a ridotta suscettibilità viene costantemente monitorata da vari enti e istituzioni di ricerca internazionali i cui risultati risultano fondamentali per definire idonei protocolli per la gestione della resistenza (IRM - Insecticide Resistance Management) a livello aziendale [4]. La causa scatenante dell'insorgenza di popolazioni resistenti agli insetticidi risiede nella progressiva selezione di individui non suscettibili esercitata dai reiterati trattamenti effettuati su generazioni successive; la riduzione di questa pressione selettiva è quindi l'elemento-chiave per il contenimento del fenomeno. Questo obiettivo può essere raggiunto mediante l'impiego di opzioni di lotta alternative, 
l'ottimizzazione della lotta chimica anche attraverso l'impiego di bioinsetticidi e il mantenimento di un'adeguata rotazione di principi attivi insetticidi con diversi meccanismi di azione $[3,4]$.

\section{Considerazioni conclusive}

Le strategie e le tecniche illustrate ci indicano che sono disponibili varie opzioni per una gestione efficace ed ecosostenibile del nuovo fitofago, che agendo sui vari stadi biologici di T. absoluta vedono l'applicazione combinata di tutti gli strumenti di lotta possibili nell'ambito delle strategie di lotta integrata, ovvero: agronomiche, fisico-meccaniche, biotecniche, chimiche, biologiche. Risulta quanto mai importante, per il raggiungimento di questo obiettivo, individuare la strategia più adatta alla situazione colturale per massimizzare gli effetti di contenimento, riducendo al minimo gli effetti secondari indesiderati provocati dall'eccesso di applicazioni insetticide.

La presenza del nuovo fitofago, ad oltre dieci anni dalla sua prima segnalazione in Europa, ha segnato un punto di svolta nei programmi di difesa in orticoltura protetta, promuovendo nuove opportunità di ricerca e sviluppo di nuove e più avanzate strategie di protezione ad alta sostenibilità.

\section{Riferimenti bibliografici}

Desneux, N., Wajnberg, E., Wyckhuys, K.A.G., Burgio, G., Arpaia, S., Narváez-Vasquez, C.A., González-Cabrera, J., Ruescas, D.C., Tabone, E., Frandon, J., Pizzol, J., Poncet, C., Cabello, T., Urbaneja, A., 2010. Biological invasion of European tomato crops by Tuta absoluta: Ecology, geographic expansion and prospects for biological control. J. Pest Sci. 83(3), 197-215.

Tropea Garzia, G., Siscaro, G., Biondi, A., \& Zappalà, L. 2012, Tuta absoluta, a South American pest of tomato now in the EPPO region: Biology, distribution and damage. EPPO Bull. 42, 205-210.

Biondi, A., Guedes, R.N.C., Wan, F.H., Desneux, N. 2018, Ecology, worldwide spread, and management of the invasive south american tomato pinworm, Tuta absoluta: past, present, and future. Annu. Rev. Entomol. 63, 239-258.

Guedes, R.N.C., Roditakis, E., Campos, M.R., Haddi, K., Bielza, P., Siqueira, H.A.A., Tsagkarakou, A., Vontas, J. \& Nauen, R. 2019, Insecticide resistance in the tomato pinworm Tuta absoluta: patterns, spread, mechanisms, management and outlook. J. Pest Sci. (in press) doi:10.1007/s10340-019-01086-9.

Biondi, A., Desneux, N., Siscaro, G., Zappalà, L. 2012, Using organic-certified rather than synthetic pesticides may not be safer for biological control agents: selectivity and side effects of 14 pesticides on the predator Orius laevigatus. Chemosphere 87(7), 803-812.

Siscaro, G., Biondi, A., Haddi, K., Rapisarda, C., Tropea Garzia, G., Zappalà, L. 2013, Orientamenti di lotta integrata per il contenimento di Tuta absoluta (Meyrick) in Italia. Atti dell'Accademia Nazionale Italiana di Entomologia. Rendiconti, vol. LX, 2012, 111-124.

Abbes, K.,Chermiti, B. 2014, Propensity of three Tunisian populations of the tomato leafminer Tuta absoluta (Lepidoptera: Gelechiidae) for deuterotokous parthenogenetic reproduction. Afr. Entomol. 22(3), 538-544.

Zappalà, L., Biondi, A., Alma, A., Al-Jboory, I.J., Arnò, J., Bayram, A., Chailleux, A., ElArnaouty, A., Gerling, D., Guenaoui, Y., Shaltiel-Harpaz, L., Siscaro, G., Stavrinides, M., Tavella, L., Vercher Aznar, R., Urbaneja, A.,Desneux, N. 2013, Natural enemies of the South American moth, Tuta absoluta, in Europe, North Africa and Middle East, and their potential use in pest control strategies J. Pest Sci. 86(4), 635-647. 
Ferracini, C., Bueno, V.H.P., Dindo, M.L., Ingegno, B.L., Luna, M.G., Salas Gervassio, N.G., Sánchez, N.E., Siscaro, G., Van Lenteren, J.C., Zappalà, L., Tavella, L., 2019. Natural enemies of Tuta absoluta in the Mediterranean basin, Europe and South America. Biocontrol Sci. Techn. 29(6), 578-609.

Siscaro, G., Lo Pumo, C., Tropea Garzia, G., Tortorici, S., Gugliuzzo, A., Ricupero, M., Biondi, A., Zappalà, L. 2019, Temperature and tomato variety influence the development and the plant damage induced by the zoophytophagous mirid bug Nesidiocoris tenuis. J. Pest Sci. 92(3), 10491056.

Biondi, A., Zappalà, L., Di Mauro, A., Tropea Garzia, G., Russo, A., Desneux, N., Siscaro, G. 2016, Can alternative host plant and prey affect phytophagy and biological control by the zoophytophagous mirid Nesidiocoris tenuis? Biocontrol 61(1), 79-90.

Naselli, M., Zappalà, L., Gugliuzzo, A., Tropea Garzia, G., Biondi, A., Rapisarda, C., Cincotta, F., Condurso, C., Verzera, A., Siscaro, G. 2017, Olfactory response of the zoophytophagous mirid Nesidiocoris tenuis to tomato and alternative host plants. Arthropod-Plant Interact. 11(2), 121-131.

Naselli, M., Urbaneja, A., Siscaro, G., Jaques, J.A., Zappalà, L., Flors, V., Pérez-Hedo, M., 2016. Stage-related defense response induction in tomato plants by Nesidiocoris tenuis. Int. J. Mol. Sci. 17(8), 1210.

Zappalà, L., Siscaro, G., Biondi, A., Mollá, O., González-Cabrera, J. ,Urbaneja, A. 2012, Efficacy of sulphur on Tuta absoluta and its side effects on the predator Nesidiocoris tenuis. J. Appl. Entomol. 136(6), 401-409.

Campolo, O., Cherif, A., Ricupero, M., Siscaro, G., Grissa-Lebdi, K., Russo, A., Cucci, L.M., Di Pietro, P., Satriano, C., Desneux, N., Biondi, A., Zappalà, L., Palmeri, V., 2017. Citrus peel essential oil nanoformulations to control the tomato borer, Tuta absoluta: Chemical properties and biological activity. Sci. Rep. 7(1), 13036. 